\title{
Genome Sequence of Dengue virus 3 from the Pythium insidiosum Transcriptomes
}

\author{
Yeonhwa Jo ${ }^{1}$, Hoseong Choi ${ }^{1}$ and Won K. Cho ${ }^{1,2 *}$ \\ 1 Department of Agricultural Biotechnology, College of Agriculture and Life Sciences, Seoul National University, Seoul, \\ Republic of Korea, ${ }^{2}$ The Taejin Genome Institute, Hoengseong, Republic of Korea
}

Keywords: genome, Dengue virus 3, Pythium insidiosum, de novo assembly, single nucleotide polymorphism

\section{BACKGROUND}

Pythium species are a kind of the fungus-like oomycetes presenting in soil and aquatic environments (Latijnhouwers et al., 2003). They are regarded as serious plant pathogens, resulting in economic losses of many crops. Of known Pythium species, P. insidiosum is the only oomycete that can infect both humans and animals (Gaastra et al., 2010). P. insidiosum causes a lifethreatening infectious diseases known as pythiosis (Mendoza et al., 1993; Gaastra et al., 2010). So far, infection of $P$. insidiosum has been reported in horses, dogs, and humans in tropical and subtropical regions. $P$. insidiosum inhabits swampy areas and produces motile zoospores colonizing surfaces of water plants (Mendoza et al., 1993; Gaastra et al., 2010). Contact of zoospores with tissues of human and animal causes serious infection (Mendoza et al., 1993).

Dengue virus (DENV) causes severe visceral and central nervous system disease in humans (Martina et al., 2009). DENV belongs to the genus Flavivirus and consists of single-stranded positive-sense RNA (Chambers et al., 1990; Bai et al., 2013). Infection of DENV is mediated by two major mosquito vectors: Aedes albopictus and Aedes aegypti (Martina et al., 2009). So far, four different serotypes of DENV have been identified in tropical and subtropical regions (Bai et al., 2013). The genome of DENV is composed of a single-stranded positive-sense RNA about 10,700 nucleotides (nt) in length. The DENV genome consists of a single open reading frame (ORF) encoding three structural (C, prM, and E) and seven nonstructural (NS1, NS2A, NS2B, NS3, NS4A, NS4B, and NS5) proteins (Chambers et al., 1990).

Next generation sequencing (NGS) facilitates the identification of known viruses in various organisms (Radford et al., 2012). NGS can be applied not only in detection or diagnosis of viruses but also in metagenome-based approaches to detect unexpected disease-associated viruses and novel viruses (Adams et al., 2009; Radford et al., 2012). In addition, complete or draft viral genomes can be assembled from NGS data (Jo et al., 2015).

In this study, we identified DENV3 from $P$. insidiosum transcriptome data and assembled a nearly complete genome of DENV3. In addition, we examined single nucleotide variations (SNVs) in the P. insidiosum transcriptome, demonstrating quasispecies of DENV3.

\section{METHODS}

\section{Oomycete Material, Growth Condition, and Library Preparation for 454 Sequencing}

The $P$. insidiosum transcriptome data was obtained from the previous study (Krajaejun et al., 2014). In brief, $P$. insidiosum strain Pi-S was isolated from a Thai patient showing vascular pythiosis. The obtained $P$. insidiosum was cultured in Sabouraud dextrose broth and incubated at $28^{\circ} \mathrm{C}$ and $37^{\circ} \mathrm{C}$ for 1 week. Mycelia from each condition was harvested and washed with sterile water. Again, the 
mycelia samples were transferred to $2 \mathrm{ml}$ of microcentrifuge tube and incubated at $28^{\circ} \mathrm{C}$ and $37^{\circ} \mathrm{C}$, respectively, for $24 \mathrm{~h}$. Harvested mycelia was immediately frozen in liquid nitrogen for further study. Poly-A tailed mRNAs were isolated from total RNAs and used for cDNA library preparation. Sequencing was performed using the Genome Sequencer (GS) FLX Titanium platform (Roche Applied Sciences, Penzberg, Germany).

\section{De novo Transcriptome Assembly}

We downloaded two raw data with following accession numbers, DRR004443 and DRR004444, from DDBJ (DNA Data Bank of Japan) (https://trace.ddbj.nig.ac.jp/index_e.html). We used a workstation (two six-core CPUs and 256-GB RAM) operated using the Ubuntu 12.04.5 LTS operation system for all bioinformatics analyses in this study. Each individual FASTQ file for each library was first subjected to de novo transcriptome assembly using the Trinity program (version 2.0.2, released 22nd January 2015) with default parameters (Grabherr et al., 2011). We obtained two independent transcriptomes from the two samples (Table 1). For de novo genome assembly of DENV3, we combined two raw data sets and performed transcriptome assembly again using Trinity, Velvet programs (Zerbino and Birney, 2008), and iAssembler program (Zheng et al., 2011). After that, contigs associated with DENV3 were selected by blast search against known DENV3 reference sequences. Genome of DENV3 was manually assembled.

\section{Identification of Viruses in the $P$. insidiosum Transcriptomes}

To identify viruses in the $P$. insidiosum transcriptomes, two different approaches were used. For the first, de novo assembled contigs were blasted against complete viral reference sequences (http://www.ncbi.nlm.nih.gov/genome/viruses/). For the BLAST search, the MEGABLAST algorithm with a cut-off $e$-value of 1e-5 was applied. MEGABLAST is much faster than other sequence similarity programs and provides very reliable sequence similarities for the identification of viruses. For the second approach, all the FASTQ files were converted into FASTA files using the FASTX-Toolkit (http://hannonlab.cshl.edu/fastx_

TABLE 1 | Summary of de novo transcriptome assembly and the number of identified DENV3 associated reads and contigs.

\begin{tabular}{lcc}
\hline Index & $\mathbf{2 8}^{\circ} \mathbf{C}$ & $\mathbf{3 7}^{\circ} \mathbf{C}$ \\
\hline SRA accession No. & DRR004443 & DRR004444 \\
Total genes & 14,327 & 17,761 \\
Total transcripts & 14537 & 18049 \\
Percent GC & 59.23 & 60.29 \\
Contig N50 & 794 & 827 \\
Median contig length & 697 & 726 \\
Average contig & 751.7 & 797.18 \\
Total assembled bases & 10927464 & 14388240 \\
Total No. of reads & 177947 & 204241 \\
No. of DENV3 reads & 638 & 229 \\
No. of DENV3 contigs & 116 & 20
\end{tabular}

toolkit/) and FASTA files from each library were directly blasted against complete viral reference sequences.

\section{Examination of Single Nucleotide Variations}

To identify SNVs, all the raw data (FASTQ files) were aligned on the obtained DENV3 genome sequence using the BurrowsWheeler Aligner (BWA) with default parameters (Li and Durbin, 2009). The obtained SAM files were converted to BAM files for sorting using SAMtools (Li et al., 2009). The BAM files were sorted for SNV calling using SAMtools. Afterward, mpileup was conducted to generate the VCF format. The SNVs were then called using BCFtools implemented in SAMtools, and finally, SNVs were filtered using BCFtools. For the visualization of mapped reads on the genome, aligned SAM files were imported to the Tablet program (Milne et al., 2010).

\section{RESULTS}

\section{Identification of DENV3 in the $P$. insidiosum transcriptomes}

In a search of viruses infecting fungi in the publicly available transcriptome data, we found DENV3-associated sequences from $P$. insidiosum transcriptomes. The transcriptomes were composed of two different libraries, which were generated from two different growth conditions, $28^{\circ} \mathrm{C}$ and $37^{\circ} \mathrm{C}$. BLAST search revealed that $0.36 \%$ (638 reads) and $0.11 \%$ (229 reads) at $28^{\circ} \mathrm{C}$ and $37^{\circ} \mathrm{C}$, respectively, were sequences associated with DENV3 (Figure 1A). The portion of DENV3 associated reads at $28^{\circ} \mathrm{C}$ was more than three times that at $37^{\circ} \mathrm{C}$. This result indicates that replication of DENV3 was reduced two-fold when the temperature was increased.

\section{De novo Viral Genome Assembly for DENV3}

Many sequenced reads were associated DENV3. Therefore, we de novo assembled transcriptome of $P$. insidiosum to identify contigs associated with the DENV3 genome. BLAST search identified $116\left(28^{\circ} \mathrm{C}\right)$ and $20\left(37^{\circ} \mathrm{C}\right)$ contigs associated with DENV3 (Figure 1B; Supplementary Table S1). The longest contig was $2332 \mathrm{bp}$, while the shortest contig was $177 \mathrm{bp}$. We assembled the genome of DENV3. The genome of DENV3, referred to as isolate Pythium, was 10,671 nucleotides (nt) in length (accession number: KT424097). By BLASTN search against the NCBI nucleotide database, the DENV3 isolate Pythium was found to be highly matched to the known DENV3 isolate HN/2013/20 (KJ622192.1) with 98\% sequence identity. The DENV3 isolate $\mathrm{HN} / 2013 / 20$ was isolated from Henan, China, in 2013, indicating the possible origin of DENV3 isolate Pythium.

We mapped raw data on the genome of the obtained DENV3 isolate Pythium. As shown in Figure 1C, the number of reads mapped on the genome was much higher in the transcriptome from $28^{\circ} \mathrm{C}$ as compared to that of $37^{\circ} \mathrm{C}$ (Figure 1C).

\section{Analysis of Single Nucleotide Variations}

We examined SNVs for DENV3 in two different transcriptomes (Supplementary Table S2). From the transcriptome at $28^{\circ} \mathrm{C}$, we identified 73 SNVs including 11 InDels (Insertion and Deletions), while 23 SNVs harboring nine InDels were detected from the 
A

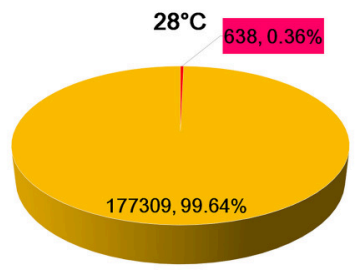

- No. of DENV reads $\quad$ No. of Pi reads

B

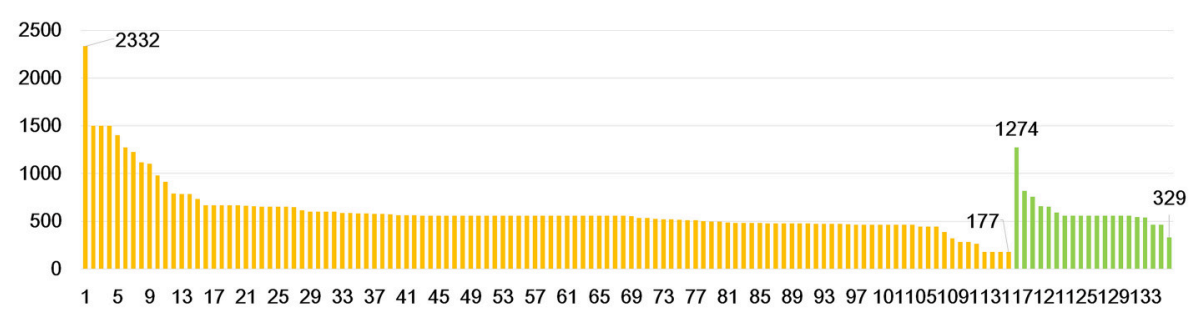

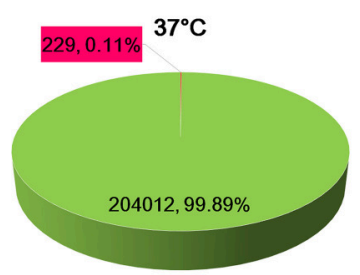

- No. of DENV reads $\quad$ No. of Pi reads
C

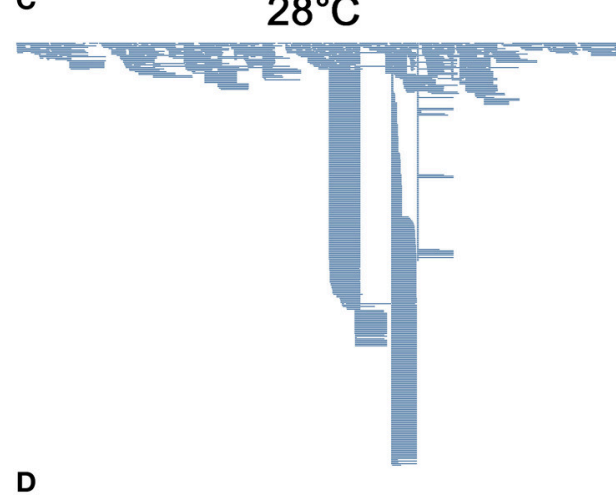

$28^{\circ} \mathrm{C}$

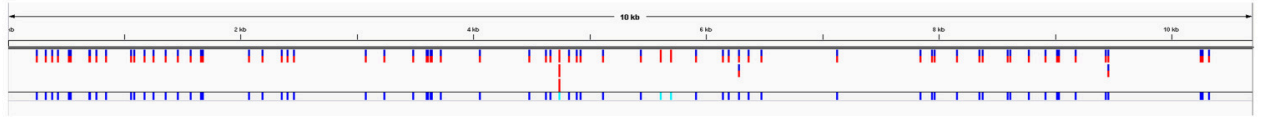

$37^{\circ} \mathrm{C}$

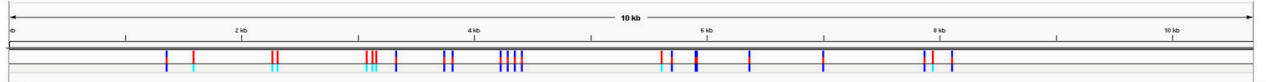

FIGURE 1 | Identification of sequences associated with DENV3 from $P$. insidiosum transcriptome data. (A) The number of reads associated with DENV3 in samples grown at $28^{\circ} \mathrm{C}$ and $37^{\circ} \mathrm{C}$ indicated by red colored box. (B) The length distribution of identified contigs associated with DENV3. Orange and green colored bars indicate samples grown at $28^{\circ} \mathrm{C}$ and $37^{\circ} \mathrm{C}$, respectively. The number indicates the length of contigs (bp) (C) Mapped DENV3 associated with reads on the assembled DENV3 genome, which was visualized by the Tablet program. (D) The locations of identified SNVs along the DENV3 genome in two samples grown at $28^{\circ} \mathrm{C}$ and $37^{\circ} \mathrm{C}$, respectively.

transcriptome at $37^{\circ} \mathrm{C}$ (Figure 1D). This data strongly supports the quasispecies nature of DENV3 within the $P$. insidiosum host (Kurosu, 2011).

\section{DISCUSSION}

The host ranges of viruses were numerous, from single cellular organisms to multicellular organisms. Previously, several viruses have been identified in many oomycetes, including Phytophthora and Pythium species (Gillings et al., 1993; Cai and Hillman, 2013). In addition, double-stranded RNA virus has been identified in the human pathogenic fungus Blastomyces dermatitidis (Kohno et al., 1994). A recent study using electron microscopy demonstrated that several human pathogenic fungi were infected by virus-like particles (Sharma et al., 2011). However, to our knowledge, no study has 
clearly identified a virus in the human pathogenic fungus so far.

To clearly demonstrate the presence of DENV3 in $P$. insidiosum transcriptomes, we assembled the genome of DENV3 isolate Pythium and examined its SNVs using Pythium transcriptome data. However, we are not sure whether the $P$. insidiosum was really infected by DENV3. It seems that the contamination rate of DENV3 in P. insidiosum culture should be very high. For example, a patient was infected with dengue virus and had viremia. Total RNAs from the viremia were transferred into $P$. insidiosum culture medium and slowly degraded during culture. Libraries were prepared from the culture medium containing dengue virus and sequenced by a next-generation sequencer. Moreover, the low amount of DENV3 at $37^{\circ} \mathrm{C}$ as compared to that at $28^{\circ} \mathrm{C}$ indicates the instability of the DENV3 RNA genome at higher temperatures. If DENV3 can replicate in $P$. insidiosum, we can get a high level of DENV3 RNA genome at $37^{\circ} \mathrm{C}$, because flavivirus polymerase usually works better at higher temperatures (Simon et al., 2015).

Many previous studies have also reported microbial contamination in clinical samples by NGS. For example, substantial bacterial contamination has routinely been identified in RNA-Seq data (Strong et al., 2014) as well as DNA data (Laurence et al., 2014). Due to the high level of sensitivity of the NGS technique, NGS is currently used to discover new viruses associated with diseases in clinical virology (Datta et al., 2015; Perlejewski et al., 2015). Taken together, this study provides the complete genome of DENV3 from $P$. insidiosum transcriptomes that might have been contaminated during sample preparation.

\section{REFERENCES}

Adams, I. P., Glover, R. H., Monger, W. A., Mumford, R., Jackeviciene, E., Navalinskiene, M., et al. (2009). Next-generation sequencing and metagenomic analysis: a universal diagnostic tool in plant virology. Mol. Plant Pathol. 10, 537-545. doi: 10.1111/j.1364-3703.2009.00545.x

Bai, Z., Liu, L.-C., Jiang, L.-Y., Liu, Q., Cao, Y.-M., Xu, Y., et al. (2013). Complete genome sequence of dengue virus serotype 3 from Guangzhou, China. Genome Announc. 1:e0020812. doi: 10.1128/genomeA.00 208-12

Cai, G., and Hillman, B. I. (2013). Phytophthora viruses. Adv. Virus Res. 86, 327-350. doi: 10.1016/B978-0-12-394315-6.00012-X

Chambers, T. J., Hahn, C. S., Galler, R., and Rice, C. M. (1990). Flavivirus genome organization, expression, and replication. Annu. Rev. Microbiol. 44, 649-688. doi: 10.1146/annurev.mi.44.100190.003245

Datta, S., Budhauliya, R., Das, B., and Chatterjee, S. (2015). Next-generation sequencing in clinical virology: discovery of new viruses. World J. Virol. 4:265. doi: 10.5501/wjv.v4.i3.265

Gaastra, W., Lipman, L. J., De Cock, A. W., Exel, T. K., Pegge, R. B., Scheurwater, J., et al. (2010). Pythium insidiosum: an overview. Vet. Microbiol. 146, 1-16. doi: 10.1016/j.vetmic.2010.07.019

Gillings, M., Tesoriero, L., and Gunn, L. (1993). Detection of double-stranded RNA and virus-like particles in Australian isolates of Pythium irregulare. Plant Pathol. 42, 6-15. doi: 10.1111/j.1365-3059.1993.tb01466.x

\section{DATA ACCESS}

The two raw data in this study can be available from DDBJ (DNA Data Bank of Japan) (https://trace.ddbj.nig.ac.jp/index_e.html) with following accession numbers, DRR004443 and DRR004444. Genome sequence of DENV3 isolate Pythium was deposited in GenBank with accession number KT424097.

\section{AUTHOR CONTRIBUTIONS}

WC designed the research; YJ, HC, and WC performed the research; YJ, HC, and WC analyzed the data; and YJ, HC, and WC wrote the paper.

\section{ACKNOWLEDGMENTS}

This work was carried out with the support of the "Cooperative Research Program for Agriculture, Science and Technology Development (Project No. PJ01186102)" conducted by the Rural Development Administration, Republic of Korea. This work is dedicated to the memory of my father, Tae Jin Cho (1946-2015).

\section{SUPPLEMENTARY MATERIAL}

The Supplementary Material for this article can be found online at: http://journal.frontiersin.org/article/10.3389/fmicb. 2016.00926

Supplementary Table S1 | Detailed information of megablast results to identify contigs associated with DENV3.

Supplementary Table S2 | Information of identified single nucleotide variations for DENV3 in two samples grown at $28^{\circ} \mathrm{C}$ and $37^{\circ} \mathrm{C}$, respectively.

Grabherr, M. G., Haas, B. J., Yassour, M., Levin, J. Z., Thompson, D. A., Amit, I., et al. (2011). Full-length transcriptome assembly from RNA-Seq data without a reference genome. Nat. Biotechnol. 29, 644-652. doi: 10.1038/nbt.1883

Jo, Y., Choi, H., and Cho, W. K. (2015). De novo assembly of a bell pepper endornavirus genome sequence using RNA sequencing data. Genome Announc. 3, e00061-e00015. doi: 10.1128/genomeA.00061-15

Kohno, S., Fujimura, T., Rulong, S., and Kwon-Chung, K. (1994). Double-stranded RNA virus in the human pathogenic fungus Blastomyces dermatitidis. J. Virol. 68, 7554-7558.

Krajaejun, T., Lerksuthirat, T., Garg, G., Lowhnoo, T., Yingyong, W., Khositnithikul, R., et al. (2014). Transcriptome analysis reveals pathogenicity and evolutionary history of the pathogenic oomycete Pythium insidiosum. Fungal Biol. 118, 640-653. doi: 10.1016/j.funbio.2014.01.009

Kurosu, T. (2011). Quasispecies of dengue virus. Trop. Med. Health 39, 29. doi: 10.2149/tmh.2011-S02

Latijnhouwers, M., De Wit, P. J., and Govers, F. (2003). Oomycetes and fungi: similar weaponry to attack plants. Trends Microbiol. 11, 462-469. doi: 10.1016/j.tim.2003.08.002

Laurence, M., Hatzis, C., and Brash, D. E. (2014). Common contaminants in nextgeneration sequencing that hinder discovery of low-abundance microbes. PLoS ONE 9:e97876. doi: 10.1371/journal.pone.0097876

Li, H., and Durbin, R. (2009). Fast and accurate short read alignment with Burrows-Wheeler transform. Bioinformatics 25, 1754-1760. doi: 10.1093/bioinformatics/btp324 
Li, H., Handsaker, B., Wysoker, A., Fennell, T., Ruan, J., Homer, N., et al. (2009). The sequence alignment/map format and SAMtools. Bioinformatics 25 , 2078-2079. doi: 10.1093/bioinformatics/btp352

Martina, B. E., Koraka, P., and Osterhaus, A. D. (2009). Dengue virus pathogenesis: an integrated view. Clin. Microbiol. Rev. 22, 564-581. doi: 10.1128/CMR. 00035-09

Mendoza, L., Hernandez, F., and Ajello, L. (1993). Life cycle of the human and animal oomycete pathogen Pythium insidiosum. J. Clin. Microbiol. 31, 2967-2973.

Milne, I., Bayer, M., Cardle, L., Shaw, P., Stephen, G., Wright, F., et al. (2010). Tablet-next generation sequence assembly visualization. Bioinformatics 26, 401-402. doi: 10.1093/bioinformatics/btp666

Perlejewski, K., Popiel, M., Laskus, T., Nakamura, S., Motooka, D., Stokowy, T., et al. (2015). Next-generation sequencing (NGS) in the identification of encephalitis-causing viruses: unexpected detection of human herpesvirus 1 while searching for RNA pathogens. J. Virol. Methods 226, 1-6. doi: 10.1016/j.jviromet.2015.09.010

Radford, A. D., Chapman, D., Dixon, L., Chantrey, J., Darby, A. C., and Hall, N. (2012). Application of next-generation sequencing technologies in virology. J. Gen. Virol. 93, 1853-1868. doi: 10.1099/vir.0. 043182-0

Sharma, S., Gupta, S., and Shrivastava, J. (2011). Presence of virus like particles in human pathogenic fungi: chrysosporium sps and Candida albicans. Indian J. Virol. 22, 104-110. doi: 10.1007/s13337-011-0048-x
Simon, A. Y., Sutherland, M. R., and Pryzdial, E. L. (2015). Dengue virus binding and replication by platelets. Blood 126, 378-385. doi: 10.1182/blood-2014-09598029

Strong, M. J., Xu, G., Morici, L., Bon-Durant, S. S., Baddoo, M., Lin, Z., et al. (2014). Microbial contamination in next generation sequencing: implications for sequence-based analysis of clinical samples. PLoS Pathog. 10:e1004437. doi: 10.1371/journal.ppat.1004437

Zerbino, D. R., and Birney, E. (2008). Velvet: algorithms for de novo short read assembly using de Bruijn graphs. Genome Res. 18, 821-829. doi: 10.1101/gr.074492.107

Zheng, Y., Zhao, L., Gao, J., and Fei, Z. (2011). iAssembler: a package for de novo assembly of Roche-454/Sanger transcriptome sequences. BMC Bioinformatics 12:453. doi: 10.1186/1471-2105-12-453

Conflict of Interest Statement: The authors declare that the research was conducted in the absence of any commercial or financial relationships that could be construed as a potential conflict of interest.

Copyright (C) 2016 Jo, Choi and Cho. This is an open-access article distributed under the terms of the Creative Commons Attribution License (CC BY). The use, distribution or reproduction in other forums is permitted, provided the original author(s) or licensor are credited and that the original publication in this journal is cited, in accordance with accepted academic practice. No use, distribution or reproduction is permitted which does not comply with these terms. 